\title{
Long-term survival and related outcomes for hybrid versus traditional arch repair-a meta-analysis
}

\author{
Adam Chakos ${ }^{1}$, Dean Jbara ${ }^{1}$, Tristan D. Yan ${ }^{1,2,3}$, David H. Tian ${ }^{1,4}$ \\ ${ }^{1}$ The Collaborative Research (CORE) Group, ${ }^{2}$ Faculty of Medicine and Health Sciences, Macquarie University, Sydney, Australia; ${ }^{3}$ Department of \\ Cardiothoracic Surgery, Royal Prince Alfred Hospital, Sydney, Australia; ${ }^{4}$ Royal North Shore Hospital, Sydney, Australia \\ Correspondence to: Adam Chakos. The Collaborative Research (CORE) Group, Macquarie University, Sydney, Australia. \\ Email: adam.chakos@outlook.com.
}

Background: Surgical interventions for aortic aneurysm and dissection remain associated with high risk of mortality and morbidity. Advances in operative techniques have led to a variety of options for the cardiac surgeon, including endovascular and hybrid approaches. Debate remains over which of these techniques provide optimal outcomes for the patient. The present systematic review and meta-analysis aims to evaluate long term patient survival and identify short-term outcomes for conventional (open) aortic arch repair and hybrid aortic arch repair (HAR).

Methods: An electronic literature search was conducted according to predefined inclusion criteria for hybrid and conventional aortic arch repair surgery. Digitized survival data was extracted from identified studies' Kaplan-Meier curves and used to re-create individual patient data for aggregated survival analysis. Post-operative morbidity and mortality were analyzed using random-effects model meta-analysis.

Results: Nine studies were included, containing 841 hybrid arch repair and 1,182 conventional arch repair patients. Pooled Kaplan-Meier analysis of all patients demonstrated higher survival in hybrid arch repair patients than conventional arch repair patients, however, this was noted to be sensitive to results from a particular study. Overall results showed for the hybrid repair cohort, survival at 1, 2, 3, 5 and 7 years was $87 \%, 85 \%, 83 \%, 78 \%$ and $75 \%$, respectively. Survival in the conventional repair cohort at 1, 2, 3, 5 and 7 years was $84 \%, 82 \%, 80 \%, 75 \%$ and $71 \%$, respectively. Statistically significant findings from meta-analysis showed hybrid arch repair was associated with lower risk of re-operation for bleeding, while conventional arch repair was associated with reduced risk of spinal cord injury.

Conclusions: Pooled Kaplan-Meier analysis of all studies showed long-term survival outcomes for hybrid and conventional aortic arch repair patients are heterogeneous and sensitive to the results of particular studies. Superior results from particular centres and the low number of comparative studies mean that more data is required to make definitive findings with regards to the long-term survival outcomes of either procedure. Hybrid arch repair was associated with lower risk of re-operation for bleeding, while conventional arch repair was associated with lower risk of spinal cord injury. Surgeons should consider their own center's experience and patient suitability when deciding between hybrid or conventional aortic repair techniques.

Keywords: Open surgical repair; aortic arch replacement; hybrid arch repair (HAR); elephant trunk; aortic arch aneurysm

Submitted May 01, 2018. Accepted for publication May 09, 2018.

doi: $10.21037 /$ acs.2018.05.07

View this article at: http://dx.doi.org/10.21037/acs.2018.05.07 


\section{Introduction}

Disease of the aortic arch carries a high risk of mortality and treatment remains a challenge in the modern age. Despite recent improvements, surgical mortality from acute dissection remains high $(1,2)$. Contemporary improvements in perfusion techniques, perioperative care and newer surgical approaches, (such as hybrid aortic arch surgery), have helped to reduce the operative risk associated treatment of aortic disease (3).

While traditional open surgery is still considered by some as the gold standard for treatment of extensive aortic arch pathologies $(4,5)$, others have suggested hybrid techniques such as the frozen elephant trunk and aortic arch vessel debranching as more appropriate procedures for high-risk patients, where co-morbidities may contraindicate cardiopulmonary bypass and longer operative times required for traditional repair $(6,7)$. Total-endovascular techniques are also being developed and may soon be a more widely utilized treatment option $(8,9)$.

Owing to the difficulty of randomizing treatments for aortic diseases, numerous retrospective institutional series have been published for aortic arch repair. Recent systematic reviews and meta-analyses have examined these series and present differences in short-term outcomes between open and hybrid aortic arch repair (HAR) techniques (10-12) and for hybrid techniques generally $(13,14)$. This review aims to examine long-term survival outcomes from comparative studies and to conduct a metaanalysis of short-term outcomes.

\section{Methods}

\section{Literature search}

Three electronic databases (Medline, Embase and PubMed) were queried from their dates of inception till February 2018 using the search terms ("aortic arch aneurysm" OR "aortic arch") AND ("arch debranching" OR "elephant trunk" OR "endovascular aortic arch repair" OR "hybrid aortic arch repair" OR "hybrid arch repair" OR "open surgical repair" OR "open arch repair") for comparative studies which reported aortic arch surgery using open or hybrid procedures. No distinction was made regarding surgical indications or neuroprotection strategies. Studies needed to include more than 20 patients per arm and provide Kaplan-Meier time-to-event curves with at least 3 years follow-up to allow secondary analysis of survival data. The primary end-points were overall survival and early mortality. Secondary end-points were evaluated if reported by at least $50 \%$ of included studies and included transient ischaemic attack, stroke, acute kidney injury, requirement for post-operative hemodialysis and prolonged ventilation.

Non-English studies, animal or laboratory studies, reviews, case reports, editorials, letters, conference abstracts and opinions were excluded. Where multiple studies reported the same cohort, only the most up-to-date study was included. Literature search and data extraction were performed by two independent researchers (A.C. and D.J.). Differences in results were resolved by consensus. A PRISMA diagram is provided in Figure S1.

\section{Quality analysis}

The quality of included studies was evaluated using an appropriated version of the schema provided by the Canadian Institute for Health Economics (15). Studies were scored using a sixteen-point metric. Those scoring in the range $8-10$ were graded standard quality, 11-12 were graded intermediate quality and 13-14 were graded high quality.

\section{Procedural definitions}

\section{Conventional/traditional arch repair}

Traditional arch repair was defined as a procedure completed using open surgical access with no endovascular component. So-called conventional total arch replacement (CTAR) is typically performed using wholly surgical methods and involves sternotomy, followed by excision and replacement of the aortic arch with a prosthetic graft. For the classic/conventional Elephant Trunk procedure, the graft is invaginated, positioned in the distal aorta and the folded end anastomosed to the open section of distal aorta. The inside fold is then everted and anastomosed with the proximal aorta- the distal end of the graft is left free-floating in the descending aorta to be secured during a second-stage procedure $(10,16,17)$. If the second stage involves securing the trunk via endovascular intervention, then the procedure is considered to be a hybrid repair (rather than traditional repair) $(10,18,19)$.

\section{Hybrid arch repair}

HAR procedures were defined as those that involved an open repair component (requiring either conventional or minimal-access sternotomy) and an endovascular component to address the full extent of aortic pathology. 
The open and endovascular portions can be performed during a single or a two-stage procedure [where pathology distal to the prosthesis is covered by a stent or the distal portion of the graft is secured following the index procedure (20)]. Procedures where sternotomy access to the aortic arch was not required [such as left common carotid to left subclavian bypass via a subclavicular incision and subsequent endovascular exclusion of the affected aorta (21)] were not included as hybrid procedures.

Areas of the aorta affected by pathology and targeted as landing-zones for endovascular stents were defined according to the zone definitions established by Mitchell, Ishimaru and colleagues (22). Hybrid arch repair was classified according to definitions used by Bavaria and colleagues (3): hybrid type I repair: aortic arch vessels are de-branched and re-implanted on the healthy native proximal aorta, with a subsequent endovascular stent graft then placed to cover the diseased aorta; hybrid type II repair: the proximal ascending aorta is resected and replaced by a prosthetic graft, followed by arch vessel de-branching and re-implantation on the prosthetic graft, and completed by an endovascular stent inserted to cover the distal portion of the diseased aorta; hybrid type III repair: a socalled 'frozen' or stented elephant trunk is deployed during conventional arch replacement to facilitate concomitant aortic arch and proximal descending aneurysmal repair in a single stage.

For the purposes of this study, the definition of hybrid type III repair was expanded to include elephant trunk procedures that utilized an open zone 2 anastomosis of an elephant trunk graft followed by endovascular "freezing" of the trunk in the descending aorta (19).

\section{Statistical analysis}

Data from included studies was extracted by two independent researchers (A.C. and D.J.). Where data was expressed as median and interquartile range, it was converted to mean and standard deviation using the methods of Wan et al. (23). Pooled data were derived using meta-analysis of proportions or means. Outcomes for traditional and hybrid patients were compared and risk ratios (RR) calculated with $95 \%$ confidence intervals (CI). A random effects model was applied to account for betweenstudy variance that would be inevitable with patient selection, procedural and care differences not accounted for in institutional series.

Digitized Kaplan-Meier curves were aggregated using techniques developed by Guyot and colleagues for conducting secondary analysis of survival data (24). This method allows individual patient time-to-event data to be inferred from the Kaplan-Meier equations, taking into account patient numbers-at-risk and assuming constant censoring where that data is not provided. Hazard ratios are calculated from Kaplan-Meier data using the Cox proportional hazard model.

Publication bias was examined with funnel plots and also by Egger's tests. Heterogeneity amongst studies were assessed using the $\mathrm{I}^{2}$ statistic. Potential sources of heterogeneity were explored, including with the aid of an L'Abbe plot, Baujat plot and a leave-one-out sensitivity analysis conducted to identify studies contributing most to heterogeneity (assessed as $\mathrm{I}^{2}$ ) and inconsistency of effect measure (25). Meta-regression was performed using median year of patient recruitment to examine temporal influences on potential heterogeneities of results.

Two-tailed $\mathrm{P}$ values $<0.05$ were deemed as significant. All statistics were performed with Matlab R2017b [The MathWorks, Natick, Massachusetts, USA (26)], or R [R foundation for statistical computing, Vienna, Austria (27)].

\section{Results}

The literature search identified 1,268 records, of which nine satisfied the inclusion criteria and definitions for hybrid versus traditional aortic arch surgery $(6,7,18-20,28-31)$ (study details in Table S1). Two studies were identified as high quality, three as intermediate quality and four as standard quality using the adapted scoring criteria. Overall there were 2,028 patients, with 1,182 patients who received traditional/CTAR and 841 patients received hybrid arch repair. Where a study presented only pooled results, HAR and CTAR procedure numbers were taken from the Kaplan-Meier strata (29). The mean length of follow up for all studies was 3.4 years (95\% CI: 2.5-4.4 y). Kaplan-Meier maximum follow-up within included studies ranged from 4 to 12 years.

The mean age of the overall cohort was 61.8 years (95\% CI: 57.2-66.5), with 70\% males. Patient baseline characteristics are summarized in Table 1. Characteristics of patients from individual studies are recorded in Table $S 2$.

Operative data for the pooled cohort is presented in Table 2 and demonstrates that overall, cardiopulmonary bypass time and cross-clamp time were all slightly shorter in hybrid cases. Cerebral perfusion time was marginally longer for HAR than CTAR, with three studies $(28,30,31)$ 


\begin{tabular}{|c|c|c|}
\hline Characteristic & Hybrid repair, n [\% (95\% Cl)] & $\begin{array}{l}\text { Conventional repair (CTAR), n [\% } \\
(95 \% \mathrm{Cl})]\end{array}$ \\
\hline Patients & 841 & 1,182 \\
\hline Males & 560 & 761 \\
\hline Age, mean $(95 \% \mathrm{Cl})$ & $63.5(54.6 ; 72.3)$ & $59.0(54.3 ; 63.7)$ \\
\hline Hypertension & $378 / 483$ [81.8 (71.1; 89.1)] & $425 / 566$ [72.7 (58.9; 83.2)] \\
\hline Diabetes mellitus & $31 / 483[7.3(4.2 ; 12.4)]$ & $40 / 566[6.6(3.4 ; 12.6)]$ \\
\hline PVD & $64 / 496[11.4(4.0 ; 28.4)]$ & $39 / 291[5.8(1.1 ; 25.4)]$ \\
\hline COPD & $95 / 319$ [26.6 (12.7; 47.6)] & $157 / 873[14.3(6.1 ; 30.1)]$ \\
\hline Prior CVA & $47 / 564[9.2(4.9 ; 16.5)]$ & $102 / 868[12.4(9.6 ; 15.9)]$ \\
\hline Coronary disease & $80 / 464[14.9(4.5 ; 39.7)]$ & $88 / 468[11.7(5.0 ; 25.0)]$ \\
\hline Renal impairment & $69 / 610[10.1(4.8 ; 19.9)]$ & 69/993 [5.5 (2.8; 10.8)] \\
\hline Prior sternotomy, cardiac, aortic surgery & $166 / 647$ [20.1 (10.2; 35.8)] & $258 / 970[23.1(10.5 ; 43.5)]$ \\
\hline \multicolumn{3}{|l|}{ Presentation } \\
\hline Aneurysm (degenerative, atherosclerotic) & $212 / 391[70.5(48.2 ; 85.9)]$ & $350 / 579$ [51.8 (28.5; 74.3)] \\
\hline Acute dissection & $237 / 476[60.6(32.4 ; 83.2)]$ & $207 / 348$ [60.6 (32.4; 83.2)] \\
\hline Chronic dissection & $238 / 536$ [26.3 (11.4; 49.7)] & $170 / 643[37.8(16.1 ; 65.8)]$ \\
\hline
\end{tabular}

Table 2 Pooled intraoperative data

\begin{tabular}{|c|c|c|c|c|}
\hline Parameter & \multicolumn{2}{|l|}{ Hybrid repair (HAR) } & \multicolumn{2}{|l|}{ Conventional repair (CTAR) } \\
\hline Concomitant AVR, n [\% (95\% Cl)] & $117 / 631$ [15.6 (9.2; 25.4)] & - & $147 / 937[18.0(9.3 ; 31.8)]$ & - \\
\hline Concomitant CABG, n [\% (95\% Cl)] & $87 / 689[13.0(9.5 ; 17.4)]$ & - & $161 / 1,061[15.0(11.7 ; 19.0)]$ & - \\
\hline CPB time (min) & $201.1(187.8 ; 214.5)$ & $660 / 841$ & $208.9(175.6 ; 242.3)$ & $1,107 / 1,182$ \\
\hline Cerebral perfusion time (min) & $51.1(35.2 ; 67.1)$ & $559 / 841$ & $49.6(22.2 ; 76.9)$ & $866 / 1,182$ \\
\hline
\end{tabular}

Values are given as pooled mean and $95 \% \mathrm{Cl}$. The percentage was calculated using meta-analysis of proportions and means. $\mathrm{Cl}$, confidence interval; AVR, aortic valve replacement; CABG, coronary artery bypass grafting; CPB, cardiopulmonary bypass.

out of four reporting SCP use for both CTAR and HAR. It is important to note that not all hybrid procedures utilized bypass, cross-clamping or cerebral perfusion. Concomitant procedures were reported for both CTAR and HAR patients across six of nine studies $(6,7,20,28,30,31)$. Operative data for individual included studies is detailed in Table S3.
Aggregated survival data is shown in Figure 1. For the pooled hybrid cohort, survival at 1, 2, 3, 5 and 7 years was $87 \%, 85 \%, 83 \%, 78 \%$ and $75 \%$ (respectively). Survival in the conventional repair cohort at 1,2, 3, 5 and 7 years was $84 \%, 82 \%, 80 \%, 75 \%$ and $71 \%$ (respectively). Hazard ratio for survival was calculated from the overall KaplanMeier curves as favoring hybrid repair [HR 0.82 (0.69; 0.99), 


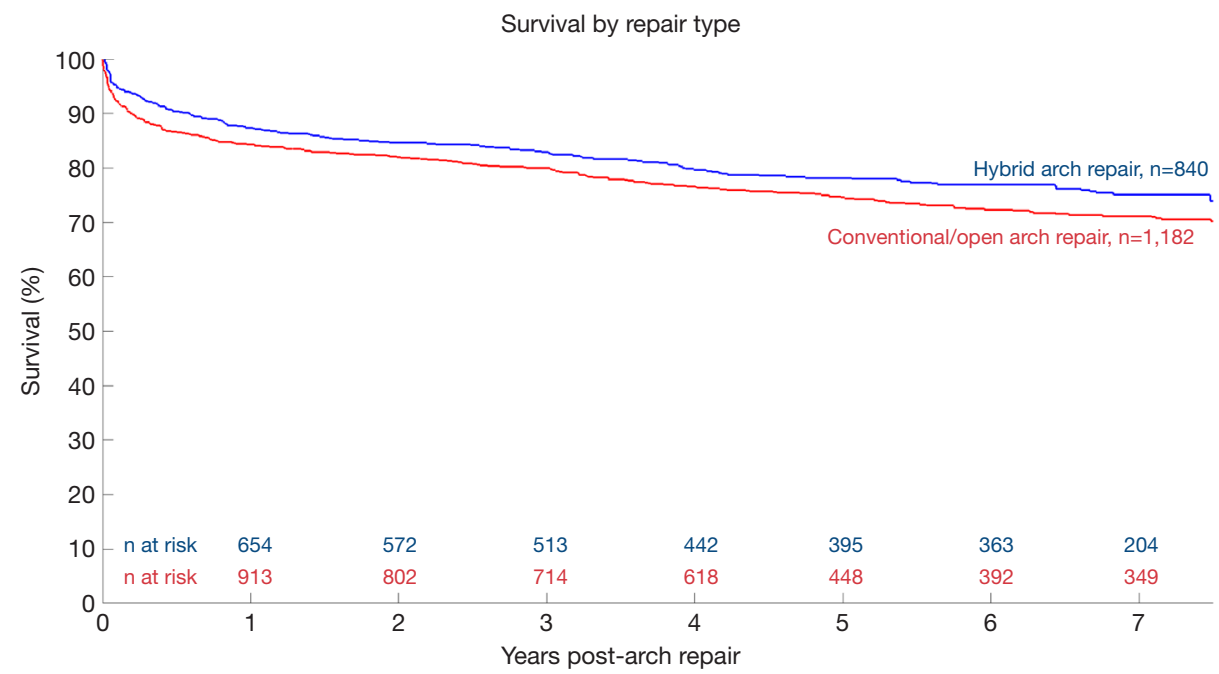

Figure 1 Kaplan-Meier survival of entire pooled cohort. Patient time-to-event data was individually calculated for each study and all patients then combined into an aggregated cohort.

$\mathrm{P}=0.04]$. Secondary analysis with a leave-one-out method found that exclusion of Sun et al. (31) changed direction of the results to favor survival in the CTAR group (HR 1.21; 95\% CI: 1.01-1.46, P=0.04, Figure S2).

Meta-analysis of outcomes (Table 3) demonstrated CTAR was associated with less spinal cord injury (RR 2.36; 95\% CI: 1.29-4.33; P<0.01, Figure 2), while a trend existed for reduced risk of reoperation for bleeding in the HAR cohort (RR 0.71; 95\% CI: 0.5-1.00, $\mathrm{P}=0.05$, Figure 3). No significant differences were seen for early mortality (Figure S3), stroke, transient ischaemic attack, acute kidney injury, requirement for post-operative hemodialysis, or prolonged ventilation (Figures $S 4-S 6$ ). Individual study outcome data is summarized in Table S4.

Meta-analysis of proportions revealed that there was significant heterogeneity for most postoperative outcomes. However, I $^{2}$ CIs (32) were often wide and sensitivity analysis demonstrated individual studies often exerted a disproportionate effect on overall/point heterogeneity [early mortality (19), transient ischaemic attack (7), acute kidney injury (7), prolonged ventilation (20)].

Meta-regression for early mortality found that the median year of patient recruitment may account for some inter-study variance $\left(\mathrm{R}^{2}=75 \%\right)$ but the meta-regression coefficient did not reach statistical significance $(\mathrm{P}=0.13)$. These findings indicate the regression is likely underpowered (Figure S7). Visual inspection of funnel plots and the results of Egger's test were examined for all studies and bias was not evident [the authors note Egger's test results may not be as robust for less than 10 studies (33)]. The funnel plot for the primary end-point is provided in Figure S8.

\section{Discussion}

The present meta-analysis pooled results from nine institutional series comparing hybrid and conventional open arch procedures. Kaplan-Meier survival analysis showed hybrid arch patients had higher survival rates than conventional arch repair patients (Figure 1) and hazard ratio for long term survival favored hybrid repair. However, these long-term survival results were sensitive to the inclusion of particular studies (Figure S2), making it difficult to draw conclusions. Secondary morbidity endpoints demonstrated conventional arch replacement was associated with lower risk of spinal cord injury, while hybrid procedures had less risk of re-intervention for bleeding. Considerable heterogeneity was identified between studies which limits the generalizability of these findings.

Individual Kaplan-Meier survival curves from included studies showed survival for CTAR above HAR for five of the nine studies, contrasting with our pooled overall survival curve (Figure 1). Close examination showed Kaplan-Meier curves from Sun (31) had significantly higher survival rates than other studies and a mean patient age 16 years younger than the mean of all other studies. Additionally, Sun et al. included hemi-arch replacement patients as part of their conventional repair cohort, a criteria other 


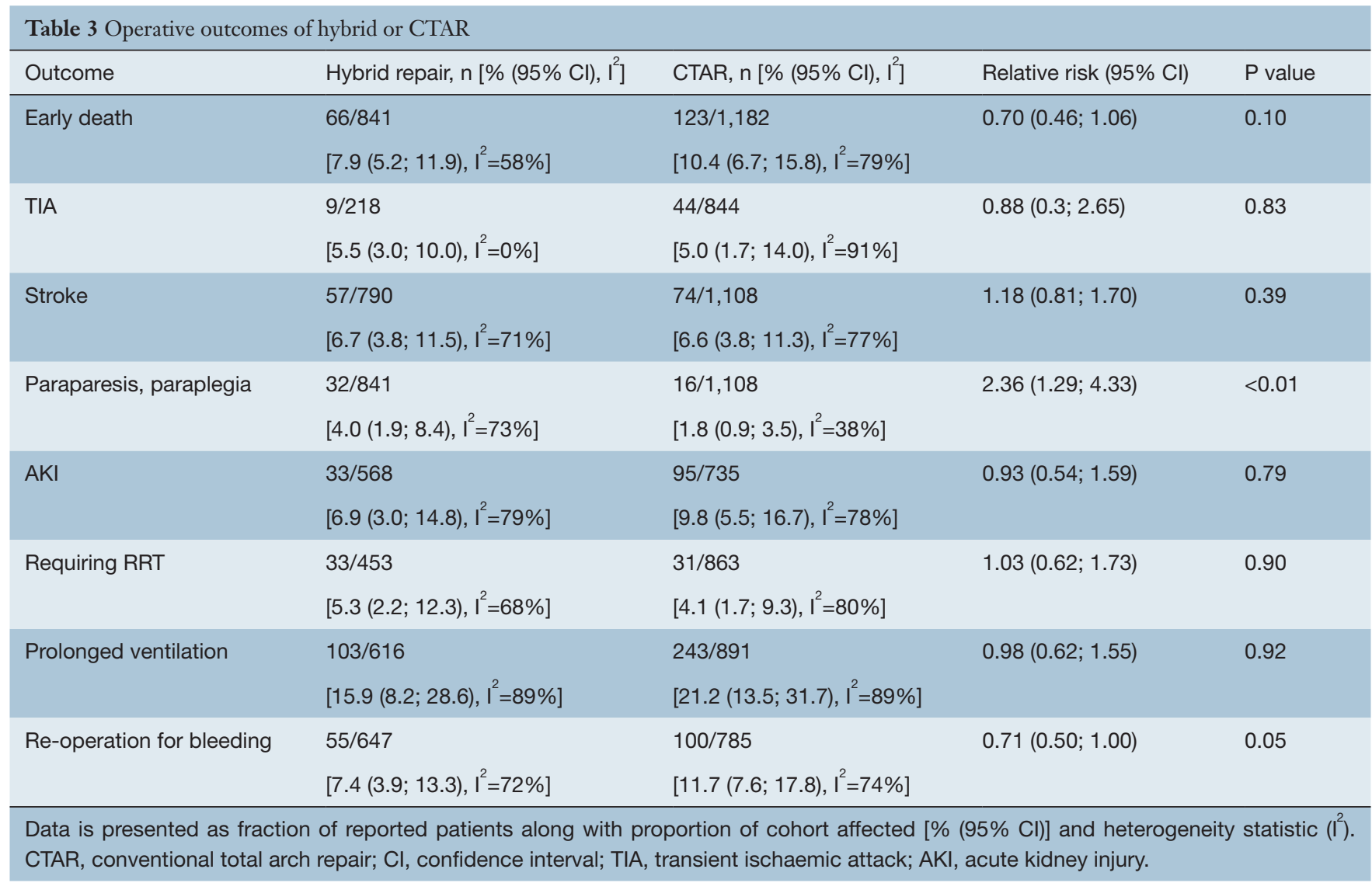

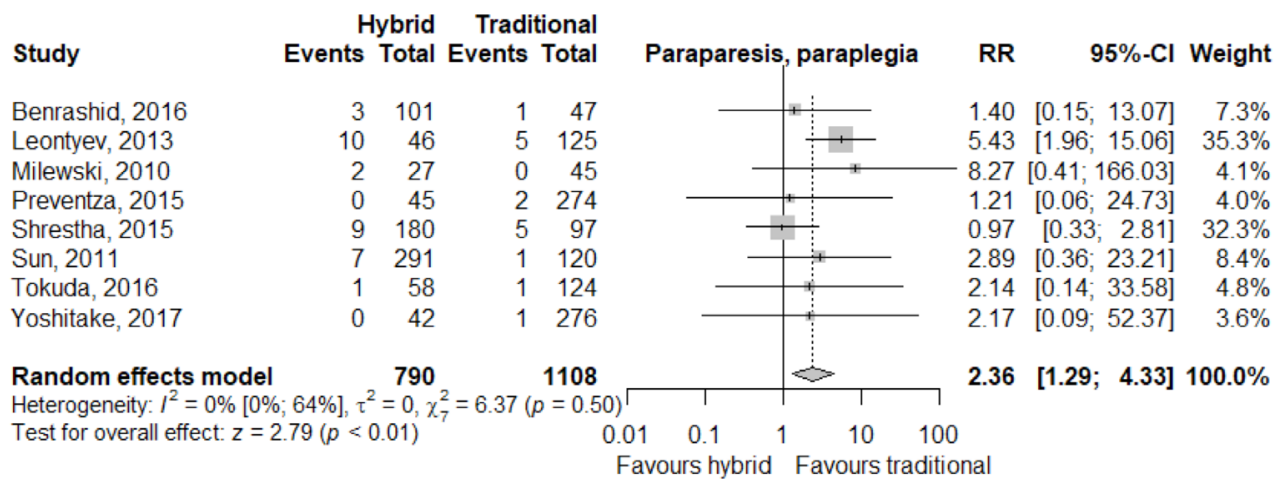

Figure 2 Forest plot for relative risk of spinal cord injury following HAR and CTAR. HAR, hybrid aortic arch repair; CTAR, conventional total aortic arch repair.

studies used as basis for exclusion (6,18-20). Given the differing baseline patient characteristics of Sun's study, pooled Kaplan-Meier analysis excluding these results was conducted, showing better long-term survival and reduced death rate for conventional repair than hybrid repair (Figure S2). The hazard ratio for long term survival also turned to favor conventional repair.

Similar to outlying long-term survival data from particular studies (31) affecting aggregated survival, individual studies also demonstrated outlying early mortality data, particularly where event rates were low or zero $(19,20)$. Contribution to heterogeneity was evidenced by a leave- 


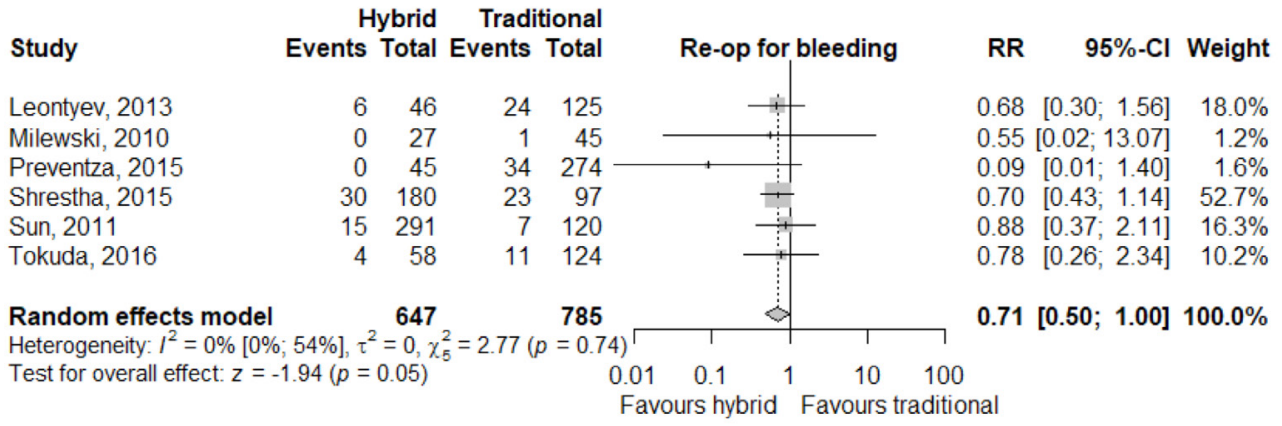

Figure 3 Forest plot for relative risk of re-operation for bleeding following HAR and CTAR. HAR, hybrid aortic arch repair; CTAR, conventional total aortic arch repair.

one-out analysis, demonstrating improved heterogeneity statistics and additionally, significance being reached for early mortality when a particular outlying study (19) was excluded [RR 0.65 (0.47; 0.91), $\mathrm{P}=0.01, \mathrm{I}^{2}=0 \%$, Figure S9]. While similar analysis for secondary (morbidity) endpoints showed potential to reduce $\mathrm{I}^{2}$ statistics (where they were not already $0 \%$ ), no change in significant findings occurred.

Meta-analysis of all studies reporting spinal cord injury showed conventional repair had a lower risk than hybrid repair, however, conventional repair had a higher relative risk of requiring re-operation for bleeding. Relative risk favoring CTAR for freedom from spinal cord injury is an acknowledged advantage $(3,28)$. Reduced rates of re-intervention for bleeding in HAR are expected given less anastomoses would be required and there is reduced requirement for cardiopulmonary bypass and/or hypothermia. Increased rates of spinal cord injury in the hybrid cohort is also unsurprising given the length of stent graft to cover aneurysmal sections of aorta may occlude intercostal artery branches arising from the false lumen. This effect may be exacerbated in acute presentations, where there has been insufficient time for collateral supply to develop $(31,34)$. Meta-analysis of early mortality and secondary outcomes of stroke, transient ischemic attacks, acute kidney injury, postoperative dialysis, and prolonged ventilation were equivocal.

While the present results agree with findings from similar analyses $(11,35)$, it is worth noting the significant degree of heterogeneity seen across almost all results. The $\mathrm{I}^{2}$ CIs were often wide and sensitivity analysis revealed heterogeneity was often largely contributed to by a minority of studies $(7,19,20)$. We believe there are several key areas that contribute to this. Firstly, a lack of consensus on procedural definitions makes between-study comparisons problematic (7). Some studies include frozen elephant trunk under CTAR and classify hybrid procedures as only hybrid type I and II (7). Others classify procedures where access to the aortic arch is purely endovascular (and supra aortic vessels are bypassed via a subclavicular incision) as hybrid (36-38). These broad definitions for conventional repair and hybrid repair may be an additional source of heterogeneity and effect attenuation-for example, one type of hybrid procedure may have a much higher risk of stroke than another hybrid procedure but the net effect is lost in pooling (21).

Attenuation of overall effect due to broad definitions was evident when examining particular meta-analytic outcomes for our included studies. For example, for early mortality, those hybrid cohorts with a large (or total) proportion of debranch procedures $(6,7,18,19)$ had RRs closer to unity than for hybrid cohorts consisting of predominantly FET patients $(28,30,31)$. Differences in intra-operative factors such as usage of cardiopulmonary bypass usage for hybrid procedures (7) or concomitant procedures potentially also lead to heterogeneity.

There were several limitations to this study, not least the suitability of only a small number of comparative studies for inclusion and the presence of many factors contributing to heterogeneity within, and between studies. In addition to possible under-powering of this study, all included studies were retrospective, institutional series. It should also be considered that hybrid procedures are a relatively recent introduction, with only a limited number of centers applying them. This not only adds learning curve as a potential confounder to the analysis, but also affects the generalizability of the findings. Additionally, included studies all utilized long, retrospective patient recruitment, with periods that averaged 10 years, reaching back to the 
turn of the century. For a dynamic and constantly evolving field such as aortic surgery, this means that poorer historical results would be included along with likely improved contemporary results, dampening their effect and increasing between-study variance. Finally, several authors describe hybrid repair patients as higher risk $(7,36)$ compared to conventional repair candidates, however, no specific risk score for aortic surgery is utilized to clearly stratify patients and establish if uniform risk cohorts are being compared (4).

\section{Conclusions}

Pooled Kaplan-Meier analysis demonstrated long-term survival in hybrid and conventional aortic arch repair patients was sensitive to the results of particular studies. Long-term survival outcomes were affected by outlying results from centers of excellence and the low number of suitable comparative studies for hybrid and conventional arch repair. Additionally, significant heterogeneity was shown to exist between studies due to multiple factors. Meta-analysis of secondary endpoints showed conventional arch replacement was associated with lower risk of spinal cord injury, while hybrid procedures had less risk of reintervention for bleeding. Surgeons should consider their individual center's experience and appropriateness of patient selection in the application of conventional or hybrid arch repair. Further studies are required to make clear findings regarding superiority of either the hybrid or conventional repair technique for long-term survival.

\section{Acknowledgements}

None.

\section{Footnote}

Conflicts of Interest: The authors have no conflicts of interest to declare.

\section{References}

1. Parikh N, Trimarchi S, Gleason TG, et al. Changes in operative strategy for patients enrolled in the International Registry of Acute Aortic Dissection interventional cohort program. J Thorac Cardiovasc Surg;153:S74-S9.

2. Boening A, Karck M, Conzelmann L, et al. German registry for acute aortic dissection type A: structure, results, and future perspectives. Thorac Cardiovasc Surg
2017;65:077-84.

3. Bavaria J, Vallabhajosyula P, Moeller P, et al. Hybrid approaches in the treatment of aortic arch aneurysms: postoperative and midterm outcomes. J Thorac Cardiovasc Surg 2013;145:S85-90.

4. Iafrancesco $M$, Ranasinghe AM, Dronavalli $V$, et al. Open aortic arch replacement in high-risk patients: the gold standard. Eur J Cardiothorac Surg 2016;49:646-51; discussion 51.

5. Khullar V, Schaff HV, Dearani JA, et al. Open Surgical Repair Remains the Gold Standard for Treating Aortic Arch Pathology. Ann Thorac Surg 2017;103:1413-20.

6. Milewski RK, Szeto WY, Pochettino A, et al. Have hybrid procedures replaced open aortic arch reconstruction in high-risk patients? A comparative study of elective open arch debranching with endovascular stent graft placement and conventional elective open total and distal aortic arch reconstruction. J Thorac Cardiovasc Surg 2010;140:590-7.

7. Preventza O, Garcia A, Cooley DA, et al. Total aortic arch replacement: A comparative study of zone 0 hybrid arch exclusion versus traditional open repair. J Thorac Cardiovasc Surg 2015;150:1591-8; discussion 8-600.

8. Yoshida RA, Kolvenbach R, Yoshida WB, et al. Total endovascular debranching of the aortic arch. Eur J Vasc Endovasc Surg 2011;42:627-30.

9. Kuratani T, Shirakawa Y, Shimamura K. Debranching TEVAR and total endovascular repair using branch devices for aortic arch pathologies. Italian Journal of Vascular and Endovascular Surgery 2015;22:5-14.

10. Di Bartolomeo R, Murana G, Di Marco L, et al. Frozen versus conventional elephant trunk technique: application in clinical practice. Eur J Cardiothorac Surg 2017;51:i20-i8.

11. Miao L, Song L, Sun SK, et al. Meta-analysis of open surgical repair versus hybrid arch repair for aortic arch aneurysm. Interact Cardiovasc Thorac Surg 2017;24:34-40.

12. Benedetto U, Melina G, Angeloni E, et al. Current results of open total arch replacement versus hybrid thoracic endovascular aortic repair for aortic arch aneurysm: A meta-analysis of comparative studies. J Thorac Cardiovasc Surg 2013;145:305-6.

13. Cao P, De Rango P, Czerny M, et al. Systematic review of clinical outcomes in hybrid procedures for aortic arch dissections and other arch diseases. J Thorac Cardiovasc Surg 2012;144:1286-300, 300.e1-2.

14. Tian DH, Wan B, Di Eusanio M, et al. A systematic review and meta-analysis on the safety and efficacy of the frozen elephant trunk technique in aortic arch surgery. 
Ann Cardiothorac Surg 2013;2:581-91.

15. Economics IoHEIEAIoH. Quality Appraisal of Case Series Studies Checklist: Institute of Health Economics (IHE) Edmonton (AB): Institute of Health Economics 2014.

16. Johnson PT, Corl FM, Black JH, et al. The elephant trunk procedure for aortic aneurysm repair: an illustrated guide to surgical technique with CT correlation. AJR Am J Roentgenol 2011;197:W1052-W9.

17. Crawford ES, Coselli JS, Svensson LG, et al. Diffuse aneurysmal disease (chronic aortic dissection, marfan, and mega aorta syndromes) and multiple aneurysm. Treatment by subtotal and total aortic replacement emphasizing the elephant trunk operation. Ann Surg 1990;211:521-37.

18. Benrashid E, Wang H, Keenan JE, et al. Evolving practice pattern changes and outcomes in the era of hybrid aortic arch repair. J Vasc Surg 2016;63:323-31.

19. Tokuda Y, Oshima H, Narita Y, et al. Hybrid versus open repair of aortic arch aneurysms: comparison of postoperative and mid-term outcomes with a propensity score-matching analysis. Eur J Cardiothorac Surg 2016;49:149-56.

20. Yoshitake A, Okamoto K, Yamazaki M, et al. Comparison of aortic arch repair using the endovascular technique, total arch replacement and staged surgerydagger. Eur J Cardiothorac Surg 2017;51:1142-8.

21. Iba $\mathrm{Y}$, Minatoya $\mathrm{K}$, Matsuda $\mathrm{H}$, et al. How should aortic arch aneurysms be treated in the endovascular aortic repair era? A risk-adjusted comparison between open and hybrid arch repair using propensity score-matching analysis. Eur J Cardiothorac Surg 2014;46:32-9.

22. Mitchell RS, Ishimaru S, Ehrlich MP, et al. First International Summit on Thoracic Aortic Endografting: roundtable on thoracic aortic dissection as an indication for endografting. J Endovasc Ther 2002;9:II-98-II-105.

23. Wan X, Wang W, Liu J, et al. Estimating the sample mean and standard deviation from the sample size, median, range and/or interquartile range. BMC Med Res Methodol 2014;14:135.

24. Guyot P, Ades A, Ouwens MJ, et al. Enhanced secondary analysis of survival data: reconstructing the data from published Kaplan-Meier survival curves. BMC Med Res Methodol 2012;12:9.

25. Anzures-Cabrera J, Higgins J. Graphical displays for metaanalysis: An overview with suggestions for practice. Res Synth Methods 2010;1:66-80.

26. Matlab release 2017b, The MathWorks Inc., Natick, Massachusetts, United States. Available online: https:// www.mathworks.com/products/new_products/ release2017b.html

27. R: A Language and Environment for Statistical Computing,
R Core Team. R Foundation for Statistical Computing, 2018. Available online: https://www.r-project.org/

28. Leontyev S, Borger MA, Etz CD, et al. Experience with the conventional and frozen elephant trunk techniques: a single-centre study. Eur J Cardiothorac Surg 2013;44:1076-82; discussion 83.

29. Nakamura K, Nagahama H, Nakamura E, et al. Predictors of early and late outcome after total arch replacement for atherosclerotic aortic arch aneurysm. Gen Thorac Cardiovasc Surg 2014;62:31-7.

30. Shrestha M, Beckmann E, Krueger H, et al. The elephant trunk is freezing: The Hannover experience. J Thorac Cardiovasc Surg 2015;149:1286-93.

31. Sun L, Qi R, Zhu J, et al. Total arch replacement combined with stented elephant trunk implantation: a new "standard" therapy for type a dissection involving repair of the aortic arch? Circulation 2011;123:971-8.

32. Ioannidis JP, Patsopoulos NA, Evangelou E. Uncertainty in heterogeneity estimates in meta-analyses. BMJ 2007;335:914-6.

33. Schwarzer G. Meta: An R package for Meta analysis. R News 2007;7:40-5.

34. Awad H, Ramadan ME, El Sayed HF, et al. Spinal cord injury after thoracic endovascular aortic aneurysm repairLésion de la moelle épinière après réparation endovasculaire d'un anévrisme de l'aorte thoracique. Can J Anaesth 2017;64:1218-35.

35. Smith HN, Boodhwani M, Ouzounian M, et al. Classification and outcomes of extended arch repair for acute Type A aortic dissection: A systematic review and meta-analysis. Interact Cardiovasc Thorac Surg 2017;24:450-9.

36. Hiraoka A, Chikazawa G, Tamura K, et al. Clinical outcomes of different approaches to aortic arch disease. J Vasc Surg 2015;61:88-95.

37. Kang WC, Ko YG, Shin EK, et al. Comparison of hybrid endovascular and open surgical repair for proximal aortic arch diseases. Int J Cardiol 2016;203:975-9.

38. Sood V, Patel HJ, Williams DM, et al. Open and endovascular repair of the nontraumatic isolated aortic arch aneurysm. J Vasc Surg 2014;60:57-63.

Cite this article as: Chakos A, Jbara D, Yan TD, Tian DH. Long-term survival and related outcomes for hybrid versus traditional arch repair-a meta-analysis. Ann Cardiothorac Surg 2018;7(3):319-327. doi: 10.21037/acs.2018.05.07 